\title{
Crenças irracionais, ajustamento psicológico e satisfação de vida em estudantes universitários
}

\author{
Adriana da Matta - Universidade Federal do Rio Grande do Sul, Porto Alegre, Brasil \\ Lisiane Bizarro - Universidade Federal do Rio Grande do Sul, Porto Alegre, Brasil \\ Caroline Tozzi Reppold - Universidade Federal de Ciências da Saúde de Porto Alegre, Brasil
}

\begin{abstract}
Resumo
Crenças irracionais (CIs) são interpretações ilógicas da realidade que colaboram para o desenvolvimento de perturbações emocionais. A premissa deste estudo é que as CIs podem estar relacionadas ao ajustamento emocional e à satisfação de vida. Estudantes universitários $(n=157)$, responderam ao Questionário de Crenças Irracionais, à Escala de Crenças Irracionais, à Escala Fatorial de Neuroticismo e à Escala de Satisfação de Vida. As CIs apresentaram correlações positivas com dois fatores da Escala Fatorial de Neuroticismo (Vulnerabilidade e Ansiedade) e negativa com idade e com satisfação de vida. Os resultados levam a reflexões importantes sobre a efetividade da Terapia Cognitiva, em especial da Terapia Racional Emotivo-Comportamental, para o ajustamento emocional e para a satisfação de vida dos clientes, que poderiam ser medidas indiretas de sucesso terapêutico.

Palavras-chave: crenças irracionais; neuroticismo; satisfação de vida.
\end{abstract}

\section{Irrational beliefs, psychological adjustment and life satisfaction in college students}

\begin{abstract}
Irrational beliefs (IB) are illogical interpretations of reality that contribute to the development of emotional problems. This study aims to show that irrational beliefs in college students $(n=157)$ might be related to emotional adjustment and life satisfaction. The participants have completed Irrational Beliefs Questionnaire, Neuroticism Factorial Scale and Life Satisfaction Scale. The IB showed positive correlation with two factors of the Neuroticism Factorial Scale (Vulnerability and Anxiety), and negative correlation with age and life satisfaction. The results suggest important considerations on the effectiveness of Cognitive Therapy, especially of Rational Emotive Therapy, for the emotional adjustment and life satisfaction of the clients, which could be an indirect measure of the therapeutic success.

Keywords: irrational beliefs; neuroticism; life satisfaction.
\end{abstract}

\section{Introdução}

Parte do processo da pesquisa clínica envolve a busca de indicadores de sofrimento e de sua associação com outros elementos que reforcem ou refutem sua impressão. O presente estudo pretendeu investigar as relações entre crenças irracionais, neuroticismo e satisfação de vida entre estudantes universitários, elementos que, até o presente momento, não foram estudados em interação.

\section{Crenças irracionais}

O conceito de crenças irracionais é central na TREC (Terapia Racional-EmotivoComportamental) (Rangé \& Fenster, 2004; Lega, 2002), que apresenta, entre seus pressupostos, as interpretações e as crenças que o indivíduo desenvolveu sobre si mesmo, sobre as outras pessoas e sobre o mundo em geral. Se essas

\footnotetext{
${ }^{1}$ Endereço para correspondência:

Laboratório de Psicologia Experimental, Neurociências e Comportamento (LPNeC). Rua Ramiro Barcelos, 2600 Bairro Santa Cecília - 90035-003 - Porto Alegre - RS Email: adrisa.ez@terra.com.br
}

interpretações ou crenças são ilógicas, têm pouca sustentação empírica e dificultam a obtenção das metas estabelecidas pelo indivíduo, recebem o nome de irracionais, o que não significa que não exista um raciocínio sobre o evento que as dispara, mas um raciocínio errôneo, já que se chega a conclusões errôneas. Se, pelo contrário, as interpretações ou inferências estão baseadas em dados empíricos e em uma sequência científica e lógica entre premissas e conclusões, as crenças são racionais, já que o raciocínio é correto e a filosofia básica dessa pessoa é funcional (Lega, 2002).

A origem das crenças irracionais está no próprio ser humano, que nasce tanto crédulo quanto educável e aceita toda a sorte de idéias, sentimentos e ações que seus pais e cuidadores mostram, sejam eles funcionais ou não. Mesmo quando se aceitam crenças irracionais alheias, há uma tendência a se reconstruir tal crença e mantêla ativa, mesmo que ela se manifeste apenas anos depois de aprendida. Algumas crenças irracionais nas crianças, como a idéia de que devem ser amadas ou aprovadas por todos aqueles que são significativos para ela, não são inteiramente impróprias à infância, pois as crianças realmente 
devem ser capazes de gerar vínculos com os outros para sobreviver. No entanto, quando essas idéias se mantêm na adolescência e na idade adulta, elas frequentemente geram importantes frustrações cujas consequências acabam por caracterizar o funcionamento desadaptado do indivíduo (Ellis, 2003). As pessoas aprendem essas crenças em seu meio social, embora tenham uma tendência inata para construir crenças irracionais a partir de suas experiências (Rangé \& Fenster, 2004). Assim, uma crença irracional, além dos limites de uma simples preferência, é uma exigência dogmática, inflexível, que colabora fortemente para o desenvolvimento de perturbações emocionais.

A partir da consideração das imposições absolutistas e das distorções cognitivas, Ellis listou 11 crenças irracionais que representam o aspecto central de seu tratamento (Rangé \& Fenster, 2004). Exemplos dessas crenças são "existe uma necessidade extrema de ser humano adulto ser amado ou aprovado por outra pessoa significativa em sua comunidade" e "é mais fácil evitar do que enfrentar certas dificuldades ou responsabilidades da vida".

O pragmatismo da psicologia logo levou à necessidade da criação de um instrumento que tornasse concreta a manifestação das crenças irracionais para o paciente; afinal, conforme Ellis (2003) considera, quando as pessoas se dão conta de que estão se sentindo mal em função de suas crenças absolutistas sobre si mesmos, sobre os outros e sobre o mundo, elas podem quase sempre debater e desafiar essas filosofias disfuncionais, indo contra elas e retornando a um estado não funcional de preferências e desejos.

De acordo com a literatura internacional, existem diversos instrumentos de avaliação das crenças irracionais, como o Survey of Personal Beliefs (Ziegler \& Smith, 2004), o Rational Behavior Inventory (Shorkey \& Whiteman, 1977) e o Attitudes and Beliefs Scale II (DiLorenzo, David \& Montgomery, 2007). Yoshida e Colugnati (2002) apresentaram dois instrumentos específicos para a verificação das crenças irracionais, com todos os critérios psicométricos necessários para sua utilização. Esses autores optaram por validar para a população brasileira dois instrumentos para avaliação de crenças irracionais, a Escala de Crenças Irracionais (ECI) originalmente desenvolvida por Malouff e Schutte (1986), com boas propriedades psicométricas, e o Questionário de Crenças Irracionais, originalmente desenvolvido por Newmark, Frerking, Cook e Newmark (1973), que, embora não com tão boas propriedades psicométicas (com carência de validade discriminante), já vinha sendo usado no contexto clínico.

A mudança mais interessante e duradoura para os pacientes envolve a reestruturação filosófica das crenças irracionais, podendo ser uma mudança específica ou geral. $O$ modelo $A B C$ da perturbação emocional de Ellis $(A=$ acontecimento ativador; $\mathrm{B}=$ crença; $\mathrm{C}=$ consequências nos sentimentos e nos comportamentos) já foi confirmado e fortalecido por diversos estudos (Hart, Turner, Hittner, Cardozo \& Paras, 1991; Ziegler \& Leslie, 2003; Ziegler \& Smith, 2004; David, Montgomery, Macavei \& Bovbjerg, 2004). Em um processo de psicoterapia acrescenta-se ainda o "D" (Rangé, 2001), que representa o combate das crenças irracionais mediante métodos lógico-empíricos da ciência, no qual a verificação de características a partir de instrumentos, como os validados por Yoshida e Colugnati (2002), se torna sobremaneira útil.

De fato, o sistema de crenças concebido e desenvolvido por Albert Ellis se tornou o objeto legítimo de análise terapêutica (Watson, Sherbak \& Morris, 1998) e, por essa razão, se faz necessário conhecer sempre mais sobre as crenças irracionais, principalmente a forma como elas podem ser acessadas, bem como as relações que se estabelecem entre as crenças irracionais e outras variáveis relacionadas à saúde mental. A importância clínica do estudo e da pesquisa das crenças irracionais se legitima na medida em que elas são eixos propulsores para a instalação de desconforto psicológico e para o desenvolvimento de psicopatologias. Conforme já mencionado, se as crenças irracionais não forem descobertas e abandonadas, os pacientes muito provavelmente continuarão mantendo-as e desenvolvendo variações irracionais. Uma questão em aberto na literatura diz respeito à forma como as crenças irracionais podem interferir no ajustamento do indivíduo e em sua instabilidade emocional.

\section{Neuroticismo}

Entre os fatores potencialmente relacionados às crenças irracionais estão os indicadores de neuroticismo. Neuroticismo é definido por Hutz e Nunes (2001) como o fator que se refere ao nível crônico de ajustamento e de instabilidade emocional, representando as diferenças individuais que ocorrem quando as pessoas experienciam padrões emocionais associados a um desconforto psicológico e os 
estilos cognitivos e comportamentais decorrentes. Hutz e Nunes (2001), em revisão da literatura, concluíram que o neuroticismo desempenha papel importante nos transtornos de personalidade, bem como nas estratégias de coping. Os dados dessa revisão mostram inclusive que escores de neuroticismo, medidos por meio de escala fatorial, têm valor preditivo em relação à ocorrência de eventos de vida estressantes, mesmo quando esses são objetivamente definidos. Os autores consideram, então, que é possível que pessoas com altos escores em neuroticismo criem ativamente problemas para elas mesmas. Considerando a natureza das crenças irracionais, com suas propriedades de tornarem catastrófico o que pode ser apenas um problema, é interessante identificar se essas estabelecem relação com fatores de neuroticismo que, conforme Hutz e Nunes (2001), incluem depressão, ansiedade, vulnerabilidade, autoestima, impulsividade e desajustamento psicossocial. Altos níveis de neuroticismo estão associados a indivíduos propensos a vivenciar mais intensamente sofrimento emocional, podendo envolver idéias dissociadas da realidade, ansiedade excessiva, dificuldade de tolerar a frustração causada pela não-saciação do desejo e respostas de coping mal adaptadas. Além disso, esses indivíduos tendem a fazer avaliações negativistas do ambiente, e a interpretar estímulos ambíguos de forma negativa ou ameaçadora, vendo ameaças, problemas e crises onde estas não existem objetivamente, o que parece mostrar associação direta com crenças irracionais.

Considerando que o neuroticismo tem papel importante nos processos adaptativos (Hutz \& Nunes, 2001) influenciando-os de forma negativa, especialmente em conjunto com as crenças irracionais, pressupõe-se uma lógica que sugere que sujeitos com considerável número de crenças irracionais ou importante carga de neuroticismo tendem, no âmbito psicológico, a sofrer mais que aqueles sem tais características. Outra pergunta em aberto na literatura diz respeito à hipótese de que a presença desses elementos tornam a percepção a respeito $\mathrm{da}$ satisfação com própria vida também caracterizada por sua presença, já que a satisfação de vida envolve uma avaliação baseada em critérios próprios, provavelmente influenciados pelas circunstâncias de vida e pelas características de personalidade dos indivíduos.

Satisfação de Vida
Satisfação de vida é o componente cognitivo de um conceito mais amplo, que é o bem-estar subjetivo. Esse componente cognitivo refere-se aos aspectos racionais e intelectuais, enquanto o componente afetivo envolve os componentes emocionais (Giacomoni, 2004). Naturalmente, as crenças irracionais estão inseridas nessa dimensão cognitiva do bem-estar subjetivo e, portanto, podem influenciá-lo, determinando a percepção dos sujeitos.

Talvez o principal indicador do bem-estar subjetivo, a satisfação de vida, corresponda à avaliação global da qualidade de vida a partir de critérios pessoalmente escolhidos, o que ocorre mediante um julgamento do quão satisfeita uma pessoa está com a sua vida atual, baseada na comparação com um padrão que cada pessoa determinou para si próprio e não um externamente imposto (Giacomoni, 2002). Assim, há um exame de aspectos reais da vida, em que são considerados aspectos positivos e negativos para a conclusão de um julgamento de satisfação de vida geral. Esse julgamento, que envolve a avaliação do bem-estar subjetivo e, portanto, da satisfação de vida, pode ser feito em diferentes domínios da vida e tem como característica a estabilidade temporal e o fato de não ser completamente dependente do estado emocional no momento da avaliação. Junto com a felicidade, que pode ser entendida como o equilíbrio entre o afeto negativo e o afeto positivo, a satisfação de vida é o elemento cognitivo, que a complementa. O bemestar subjetivo pode ser influenciado por variáveis tais como idade, gênero, nível socioeconômico e cultura, e, sendo o aspecto cognitivo do bem-estar, está diretamente ligado às crenças que se estabelecem para si, para o mundo e para o futuro (Giacomoni, 2002).

A partir do conhecimento teórico e clínico a respeito das crenças irracionais, conforme foram descritas por Albert Ellis (2003), é possível pressupor a existência de uma relação direta entre sua presença e a de fatores que representam sofrimento e desconforto psicológicos. Da mesma forma, é possível prever correlações inversas entre os elementos que predizem maior satisfação e conforto em relação à presença de um número significativo de crenças irracionais (David \& cols., 2004; Watson \& cols., 1998).

Assim, o presente estudo teve por objetivo verificar as relações entre as crenças irracionais, medidas por meio da Escala de Crenças Irracionais e do Questionário de Crenças Irracionais, os indicadores de neuroticismo, medidos por meio da 
Escala Fatorial de Neuroticismo e a avaliação de satisfação de vida, mensurada pela Escala de Satisfação de Vida. De forma específica, pretendeu-se identificar quais os fatores avaliados pelo EFN estão mais associados às crenças irracionais, bem como quais as direções de tais relações. Foram investigadas também as correlações entre as crenças irracionais e a satisfação de vida, e, por fim, se existem diferenças entre os sexos ou entre outras variáveis sociodemográficas nos escores das medidas avaliadas.

\section{Método}

\section{Participantes}

A amostra foi composta por 157 participantes, entre 17 e 49 anos (média=21 anos; $\mathrm{DP}=4,15$ anos), provindos de diversos cursos de graduação da região da grande Porto Alegre. Os cursos pesquisados foram Biomedicina, $(17,2 \%$ da amostra), Fonoaudiologia (19,1\%), Nutrição (9,6\%), Odontologia (28\%) e Psicologia (26,1\%). Não houve diferenças significativas entre as médias dos resultados dos instrumentos quando comparados os diversos cursos de graduação $(\mathrm{F}=1,16 ; \mathrm{gl}=4 ; \mathrm{p}=0,32)$. Quanto à distribuição entre os sexos, $23,2 \%$ eram do sexo masculino e $76,8 \%$ do sexo feminino. No que diz respeito ao estado civil, 3,2\% dos participantes vivem com companheiro, $39,6 \%$ afirmam não ter companheiro. Por ser uma amostra de universitários, ofereceu-se a opção "namorando", que foi apontada por $57 \%$ dos sujeitos. Entre os homens, 43\% namoram, $6 \%$ são casados e $51,4 \%$ não têm companheiro/a. Das participantes do sexo feminino, $61,3 \%$ namoram, $2,5 \%$ são casadas e $36,1 \%$ não tem companheiro/a. Como a pesquisa foi realizada entre universitários, apenas $1,9 \%$ dos participantes já tinha concluído um curso superior antes, e 2,6\% tinham, além do curso superior, pós-graduação.

A seleção da amostra utilizou critério de conveniência. O tamanho de amostra contemplou a necessidade de participantes para que se obtivesse uma probabilidade de erro do Tipo 1 de 0,05 e uma probabilidade de erro do Tipo 2 de $20 \%$ ou power de 0,80 quando alfa $<0,05$.

A coleta de dados teve início após o projeto ter sido aprovado pelo Comitê de Ética em Pesquisa da UFRGS em ofício 2007/033. A rotina da coleta de dados consistia na busca pela oportunidade autorizada de apresentação da pesquisa e subsequente administração dos instrumentos aos alunos em salas de aula. A participação dos alunos foi voluntária, sendo solicitada a ciência e a assinatura do Consentimento Livre e Esclarecido, elaborado conforme as orientações do Conselho Nacional de Saúde (Resolução 196/1996) e do Conselho Federal de Psicologia (Resolução 16/2000) no que diz respeito à ética na pesquisa com seres humanos. Os instrumentos eram apresentados e descritos, sendo solicitado aos participantes, então, respondê-los com o máximo de concentração, sinceridade e espontaneidade. Os testes foram administrados em um único momento com cada grupo de alunos. Tanto durante a coleta quanto durante a análise dos dados foram tomadas providências para garantir o anonimato e a confidencialidade dos participantes.

\section{Instrumentos}

Ficha de dados sociodemográficos: A ficha de dados sociodemográficos investigou a idade, o sexo, estado civil e a escolaridade dos participantes.

Questionário de Crenças Irracionais: Este instrumento é composto por 11 afirmações referentes às crenças irracionais na forma como foram propostas por Albert Ellis. Para cada afirmação o sujeito deve escolher entre a alternativa verdadeira ou falsa, e quanto mais respostas positivas às afirmações, mais irracional será o funcionamento emocional do indivíduo e, por conseguinte, mais comprometida sua adaptação (Yoshida \& Colugnati, 2002). O questionário foi criado por Newmark e cols. em 1973 e validado no Brasil por Yoshida e Colugnati (2002). No estudo de validação desse instrumento para a população brasileira, a análise de consistência interna realizada mediante correlação Tetracórica apresentou um índice de 0,71. O índice de precisão de teste-reteste obtido foi 0,72 .

Escala de Crenças Irracionais: Esta escala foi criada por Malouff e Schutte, (1986), sendo composta por 20 itens, aos quais os sujeitos devem responder por meio de uma escala likert de 5 pontos, cujas âncoras variam de discordo fortemente (1) a concordo fortemente (5). Esta escala pode variar entre 20 e 100 pontos e, quanto mais alto, mais crenças irracionais. No estudo de validação do instrumento para o Brasil, realizado por Yoshida e Colugnati (2002), as análises fatoriais resultaram na extração de 16 itens agrupados em um único fator. A variância explicada pelo fator é $18,87 \%$. O alfa de Cronbach 
obtido foi de 0,73 . O índice de precisão testereteste da escala foi 0,88 para a amostra masculina e 0,82 para a amostra feminina. A validação brasileira do instrumento foi realizada com estudantes universitários. Não há estudos para identificação do ponto de corte na população brasileira.

Escala Fatorial de Neuroticismo: desenvolvida por Hutz e Nunes em 2001, a EFN é um instrumento que avalia ajustamento emocional, segundo o modelo dos Cinco Grandes Fatores de Personalidade. É composto por 82 itens agrupados em quatro subescalas. A subescala de Vulnerabilidade é composta por itens que descrevem medo de críticas, insegurança, dependência às pessoas mais próximas, baixa autoestima e dificuldades de tomar decisões. Altos escores nesse fator revelam indivíduos que tendem a ser agressivos e hostis com as pessoas, podendo mentir e manipular as situações para se beneficiar. Pode indicar padrões elevados de consumo de álcool, tendência à prática de jogos de azar, e grande preocupação com a aparência física. Também são encontradas pessoas que tendem ao gosto pelo envolvimento em situações perigosas e têm pouca preocupação com o sofrimento dos demais, com as regras sociais, podendo se envolver em infrações graves (Hutz \& Nunes, 2001). A subescala de Desajustamento Psicossocial contém itens que descrevem comportamentos sexuais de risco ou atípicos, consumo exagerado de álcool, hostilidade com pessoas ou animais, tendência à manipulação de pessoas e descaso com regras sociais. Altos escores em Desajustamento Psicossocial revelam indivíduos que tendem a ser muito agressivos e hostis com os demais, podendo ser pouco sensíveis ao sofrimento alheio, e apresentar pouca preocupação com regras sociais. A sub-escala de Ansiedade agrupa itens que descrevem sintomas somáticos de transtornos relacionados à ansiedade, como irritabilidade, transtornos do sono, impulsividade, sintomas de pânico e mudanças de humor. Altos escores nesta escala indicam instabilidade emocional, variação de humor e de disposição. Há irritabilidade, medo de perder o controle das situações, fuga de idéias em função de um ritmo de pensamento acelerado e desconexo. Os desdobramentos dos transtornos de ansiedade, como os sintomas de pânico, fobias e dificuldades de concentração também são descritos nessa escala, principalmente quando há altos escores. Escores baixos podem indicar falta de motivação ou de habilidades de adaptação, pois há tendência de exposição a situações que podem oferecer risco físico ou psicológico (Hutz \& Nunes, 2001). A subescala de Depressão agrupa itens relacionados com escalas de depressão, ideação suicida e desesperança, apresentando itens que descrevem pessimismo, sentimentos de solidão e de falta de objetivos na vida. Escores altos nessa escala indicam baixas expectativas em relação ao futuro, e percepções de que a vida é monótona ou sem emoção, podendo ou não levar à ideação suicida. Já escores muito baixos permitem inferir nível baixo de autocrítica, bem como passividade em relação aos eventos que ocorrem em suas vidas (Hutz \& Nunes, 2001).

As soluções fatoriais encontraram que $\mathrm{O}$ fator vulnerabilidade obteve eigenvalues de 19,20; o fator desajustamento, de 5,12; o fator ansiedade, de 4,35 e o fator depressão, de 3,09. O alfa de Cronbach obtido no fator vulnerabilidade foi 0,89 ; no fator desajustamento psicossocial foi 0,82 , no fator ansiedade foi 0,87 e no fator depressão foi 0,87. Alfa de Cronbach na escala total foi 0,94 .

Escala de Satisfação de Vida: Criada por Diener, Emmons, Larsen e Griffin (1985) e adaptada por Giacomoni e Hutz (1997) também com boas propriedades psicométricas, consiste em uma escala tipo likert de sete pontos, composta por cinco itens relativos à percepção de satisfação de vida, e pode ser utilizada tanto com jovens quanto com adultos e idosos. Estudos confirmaram a validade concorrente e discriminante do instrumento, bem como sua consistência interna, com alfa de Cronbach de 0,82. A avaliação é somatória. Para avaliação dos escores por faixas, as classificações são: a) 35 a 31, extremamente satisfeito; b) 26 a 30 satisfeito; c) 21 a 25 , moderadamente satisfeito; d) 20, neutro; e) 15 a 19, moderadamente insatisfeito; f) 10 a 14 , insatisfeito; e g) 5 a 9, extremamente insatisfeito (Diener \& cols., 1985).

\section{Resultados}

\section{Análises descritivas}

Análises descritivas realizadas demonstram a média obtida na Escala de Crenças foi 58,3 $(\mathrm{DP}=11,31)$. No presente estudo, a Escala de Crenças revelou índice de precisão (alfa de Cronbach) de 0,78 , mostrando boa consistência interna do instrumento. Não houve diferenças significativas entre as médias nos resultados desse instrumento quando comparados homens e mulheres $(t=-0,43, \mathrm{gl}=150, p=0,6)$. O item que 
obteve maior percentual de respostas indicando total concordância dos participantes foi aquele que descreve a crença de que "para ser feliz preciso ser amado pelas pessoas que são importantes para mim". O escore 5 foi marcado por $41 \%$ dos participantes nesse item e $34,4 \%$ no item "detesto quando não posso eliminar uma dúvida”.

O Questionário de Crenças Irracionais, por sua natureza dicotômica, não pode ser avaliado conforme os métodos tradicionais de análise fatorial utilizados nos demais instrumentos. Sua interpretação parte do princípio que quanto maior o escore, maior a presença de crenças irracionais. Como no caso da Escala de Crenças, não existem estudos para identificação do ponto de corte na população brasileira para esse instrumento. Pela experiência de clínicos-pesquisadores, um resultado superior a sete já é suficiente para indicar uma condição clínica Yoshida, comunicação pessoal). A média da população estudada foi $3,30 \quad(\mathrm{DP}=1,7 ; \quad$ mínimo $=0$, máximo=8). Não houve diferenças significativas entre os sexos para esse instrumento $(t=0,38$, $\mathrm{gl}=150, \quad p=0,9)$ nem para estado civil. Curiosamente, a crença apontada como verdadeira por $67 \%$ dos participantes (a mais apontada como verdadeira) é aquela que diz que "coisas perigosas ou ameaçadoras são causas de grandes preocupações, e a possibilidade de sua ocorrência precisa ser continuamente avaliada". Outra crença apontada como verdadeira na frequência de $63,2 \%$ é a que refere que "as experiências e os acontecimentos passados são os determinantes do comportamento atual; a influência do passado não pode ser erradicada".

A Escala Fatorial de Neuroticismo é dividida em quatro fatores, que foram avaliados individualmente. Cabe salientar que não houve diferenças significativas entre as médias dos alunos conforme o sexo em nenhuma das subescalas (F1: $t=-0,49, \mathrm{gl}=159, p=0,6 ; \mathrm{F} 2: t=0,8, \mathrm{gl}=151, p=0,4$; F3: $t=-1,8, \quad g l=152, \quad p=0,6 ; \quad \mathrm{F} 4: \quad t=1,2, \quad \mathrm{gl}=148$, $p=0,9)$. A média da escala de Vulnerabilidade (F1) gerada pelos dados dos participantes da presente pesquisa foi 77,32 (DP=25,7), com índice de precisão (para essa análise sempre definida pelo alfa de Cronbach) de 0,92, o que revela boas propriedades psicométricas. A média da escala de Desajustamento Psicossocial (F2) foi 31,31 $(\mathrm{DP}=22,84)$, com índice de precisão de 0,96. A média da escala de Ansiedade (F3) foi 77,71 $(\mathrm{DP}=29,8)$, com índice de precisão de 0,92. A média da escala de Depressão (F4) foi 49,4 $(\mathrm{DP}=28,9)$, com índice de precisão de 0,96.
Apesar de a amostra ter sido composta por uma maioria de mulheres $(76,8 \%)$, apenas a escala de satisfação de vida revelou diferenças significativas entre homens e mulheres $(t=-2,9$, $\mathrm{gl}=153, \quad p \leq 0,01)$, sendo que as mulheres mostraram maior satisfação de vida (média $=26,23$, $\mathrm{DP}=5,1$ ) do que os homens (média $=23,4$, $\mathrm{DP}=4,83$ ). Segundo os critérios de Diener e cols. (1985), as mulheres podem ser consideradas satisfeitas, enquanto os homens podem ser considerados levemente satisfeitos.

\section{Correlações}

Análises de correlação de Pearson foram realizadas, uma vez que a distribuição das variáveis foi normal. Essas análises revelaram diversas relações significativas entre os construtos avaliados. No que concerne especificamente às crenças irracionais, pode-se dizer que há uma correlação significativa negativa e fraca $(r=-0,21$; $p \leq 0,05)$ entre a idade dos participantes e a presença considerável de crenças irracionais mensuradas através do Questionário de Crenças Irracionais. Isso significa que, quanto maior a idade do participante, menos significativa é a presença de crenças irracionais conforme esse instrumento. A Escala de Crenças também levou a uma correlação negativa, embora não significativa com a idade $(r=-0,4 ; p \leq 0,6)$. A correlação obtida entre o Questionário de Crenças Irracionais e a Escala de Crenças foi significativa, positiva e moderada $(r=0,56 ; \quad p \leq 0,01)$, mostrando que avaliam construtos correlatos, mas que abrangem facetas distintas do conceito de crenças irracionais.

Em relação às escalas do EFN, as crenças irracionais (avaliadas através do Questionário de Crenças Irracionais) também se correlacionam de forma significativa, positiva e fraca $(r=0,23$; $p \leq 0,02)$ com a escala de Vulnerabilidade do EFN, mostrando que há uma tendência em, se aumentando Vulnerabilidade, aumentarem as crenças irracionais, ou vice-versa. $\mathrm{O}$ mesmo acontece com o fator Ansiedade do EFN, $(r=0,18$; $p \leq 0,01)$; quanto mais crenças irracionais medidas por esse instrumento, mais ansiedade, o que foi confirmado pela Escala de Crenças. Existe uma correlação igualmente significativa, mas positiva e fraca $(r=0,3 ; p \leq 0,01)$ entre a escala Ansiedade e as crenças irracionais, medidas através da Escala de Crenças.

Em relação à satisfação de vida, as crenças irracionais, desta feita avaliadas pela Escala de Crenças, mostram uma correlação significativa $(r=-0,28 ; p \leq 0,01)$, negativa e fraca, indicando a 
tendência em, quanto mais satisfação de vida, menos crenças irracionais. O Questionário de Crenças Irracionais apresentou uma correlação negativa com satisfação de vida, embora fraca e não significativa $(r=-015 ; p \leq 0,06)$.

Quanto aos demais instrumentos que não investigam diretamente as crenças irracionais conforme o construto de A. Ellis, há relacionamento significativo entre moderado $\mathrm{e}$ forte entre todas as escalas do EFN, e uma relação significativa, negativa e fraca entre satisfação de vida e depressão, o fator 4 do EFN.

Tabela 1. Correlações entre resultados da ECI, QCI, ESV, Fatores do EFN e Idade*

\begin{tabular}{|c|c|c|c|c|c|c|c|}
\hline & EC & QCI & ESV & EFN F1 & EFN F2 & EFN F3 & EFN F4 \\
\hline Idade & $-0,42$ & $-0,211^{* *}$ & $-0,185^{*}$ & 0,061 & $-0,076$ & 0,019 & 0,019 \\
\hline EC & & $0,567 * *$ & $-0,286 * *$ & $0,195^{*}$ & 0,036 & $0,297 * *$ & 0,075 \\
\hline QCI & & & $-0,153$ & $0,234 * *$ & 0,044 & $0,189 *$ & $-0,232$ \\
\hline ESV & & & & $-0,178 *$ & $-0,031$ & $-0,093$ & $-0,232 * *$ \\
\hline EFN F1 & & & & & $0,574 * *$ & $0,671 * *$ & $0,689 * *$ \\
\hline EFN F2 & & & & & & $0,631 * *$ & $0,718^{* *}$ \\
\hline EFN F3 & & & & & & & $0,718 * *$ \\
\hline
\end{tabular}

Quando analisadas as subamostras que obtiveram escores mais altos (+1DP) e mais baixos (-1DP) na Escala de Neuroticismo as correlações se mantiveram significativas e ainda mais fortes, em especial da EFN4 (Depressão), embora negativas tanto para a Escala de Crenças quanto para o Questionário de Crenças Irracionais. Os resultados são apresentados na Tabela 2.

Tabela 2. Correlações entre resultados ECI, QCI, ESV e fatores do EFN para subamostras com valores extremos na EFN*

\begin{tabular}{lllllll}
\hline & QCI & ESV & EFN F1 & EFN F2 & EFN F3 & EFN F4 \\
\hline EC & $0,57^{* *}$ & $-0,29 * *$ & 0,23 & $-0,49$ & $0,33^{*}$ & $-0,76^{*}$ \\
& $\mathrm{n}=153$ & $\mathrm{n}=153$ & $\mathrm{n}=49$ & $\mathrm{n}=16$ & $\mathrm{n}=48$ & $\mathrm{n}=18$ \\
QCI & $-0,16$ & 0,26 & $-0,12$ & 0,32 & $-0,77 * *$ \\
& & $\mathrm{n}=153$ & $\mathrm{n}=50$ & $\mathrm{n}=16$ & $\mathrm{n}=48$ & $\mathrm{n}=18$ \\
ESV & & 0,079 & $-0,27$ & 0,17 & $-0,65^{* *}$ \\
& & & $\mathrm{n}=51$ & $\mathrm{n}=18$ & $\mathrm{n}=49$ & $\mathrm{n}=20$ \\
\hline
\end{tabular}

${ }^{*}$ Nota: ${ }^{*} p \leq 0,05 ;{ }^{*} p \leq 0,01$ EC: Escala de Crenças; QCI: Questionário de Crenças Irracionais, ESV: Escala de Satisfação de Vida; EFN: Escala Fatorial de Neuroticismo, F1: Vulnerabilidade, F2: Desajustamento Psicossocial, F3: Ansiedade, F4: Depressão.

\section{Discussão}

O presente trabalho investigou as relações existentes entre as crenças irracionais, o neuroticismo e a satisfação de vida entre estudantes universitários. Com base em uma revisão da literatura, a expectativa inicial era de que as correlações entre os instrumentos e, portanto, entre os construtos, se mostrassem significativas e fortes. Embora significativas, a maioria das correlações se revelou fraca, exceto entre os instrumentos que investigavam construtos afins, como os que investigam as crenças irracionais, ou entre as escalas do EFN, um instrumento de boa consistência interna. No entanto, há que se partir do princípio que o presente estudo foi elaborado sobre dados de uma população normal, de universitários, e não uma população clínica. Por essa razão, procedeu-se à análise de correlações com sub-amostras de valores extremos na $\mathrm{EFN}$, o que revelou um relacionamento forte e de direção negativa entre EFN4 (depressão) e crenças irracionais.

Coerente com os pressupostos da TREC, os achados do presente estudo indicam uma tendência de que se diminuam as crenças irracionais conforme os anos vão passando. Assim, talvez a baixa média de idade entre os participantes (21,25 anos) seja responsável pelos curiosos $41 \%$ de plena aceitação de uma das crenças irracionais mais tradicionais, que versa que "para ser feliz preciso ser amado pelas pessoas que são importantes para mim". Essa crença representa a necessidade de adaptação nesta etapa 
de desenvolvimento levada ao grau da irracionalidade. Marcotte (1996) verificou a mesma tendência para a categoria de crenças específicas relativas à baixa tolerância à frustração, enquanto a categoria de crenças relativas à autoregras moralistas e extremas tenderia a aumentar com a idade.

Elementos como ansiedade e raiva estão frequentemente relacionados entre si e com as crenças irracionais, e talvez alimentem algumas das crenças irracionais. Hart, Turner, Hittner, Cardozo e Paras (1991), também em uma população universitária, testaram a hipótese de que níveis de raiva e de ansiedade fossem altos em indivíduos com altos níveis de crenças irracionais, sendo concluído nesse momento que apenas raiva mostrou um efeito de interação específico (e moderado) para altos níveis de crenças irracionais. Diferentemente desses autores, o presente estudo encontrou correlação positiva e significativa (embora fraca) entre os dois instrumentos de avaliação de crenças irracionais e a Escala de Ansiedade do EFN (F3), talvez pela forma específica como Hutz e Nunes (2001) descrevem a ansiedade, sendo o conjunto de sintomas que envolve tanto sensações corporais, como vertigem, tontura e desmaio, quanto sensações negativas de irritabilidade, perda de controle e fuga de ideias. De forma mais específica, Malouff, Schutte e McClelland (1992) identificaram que escores nas medidas de crenças irracionais foram encontrados em associação com escores de ansiedade-estado, bem como demonstraram achados de que tal relação é capaz de prever o aumento no estado de ansiedade quando são experienciadas situações estressantes. Conclusões similares são apresentadas por David e colaboradoress (2004) e DiLorenzo, David e Montgomery (2007), que mostraram que, em situações estressantes, altos escores de crenças irracionais são associados à presença de sentimentos negativos, sejam eles funcionais ou disfuncionais. Em suma, medidas de crenças irracionais podem ser utilizadas para ajudar a identificar indivíduos que tendem a se tornar muito ansiosos de forma geral e especialmente quando sob pressão (Malouff, Schutte \& McClelland, 1992).

O grau de neuroticismo, no presente estudo associado de forma significativa às crenças irracionais também na Escala de Vulnerabilidade (F1), que se refere à forma como os indivíduos vivenciam sofrimentos em decorrência da aceitação do outro, parece confirmar outros achados na literatura. Escores aumentados de crenças irracionais também estão associados na literatura à baixa autoestima (contemplado por F1), bem como à menor utilização de estratégias de coping cognitivas (também contemplado por F1), com aumento do uso da estratégia de evitação (Mayhew \& Edelmann, 1989). Conforme Hutz e Nunes (2001), o neuroticismo também desempenha papel importante no processo de coping. Warren, Zgourides e Jones (1989) confirmaram que a presença considerável de crenças irracionais poderia ser um preditor da estratégia de evitação. Por outro lado, a literatura mostra que também há indicadores de que altos escores de crenças irracionais estão associados à significativa frequência de discussões ou brigas em ambientes domésticos ou escolares (Ziegler \& Leslie, 2003); ou seja, na lógica das crenças irracionais ou do neuroticismo, não há garantias de que as estratégias de evitação de fato contribuam para a ausência de conflitos ou brigas, reiterando que, de alguma forma, pessoas que têm altos escores de neuroticismo tendem a criar problemas de forma ativa para si mesmas (Hutz \& Nunes, 2001). O mesmo pode ser estendido às crenças irracionais. DiLorenzo, David e Montomery (2007), também investigando universitários, concluíram que diversas distorções cognitivas estavam significativa e diretamente associadas aos níveis de estresse, o que confirma a hipótese de que as crenças irracionais podem estar relacionadas às habilidades adaptativas dos indivíduos. Cabe destacar que ambos os instrumentos de crenças irracionais se relacionaram de forma negativa e forte com a Escala de Depressão (EFN4), o que é apenas parcialmente consistente com a literatura. Marcotte (1996), em um estudo com adolescentes, investigou a relação entre crenças irracionais e sintomas depressivos, e, a partir da categorização das crenças irracionais, verificou que apenas duas categorias de crenças irracionais estavam aumentadas em indivíduos depressivos: crenças que demonstravam baixa tolerância à frustração e crenças relativas a autorregras moralistas e extremas. Outras categorias de crenças não se relacionaram de forma significativa com depressão. Por sua vez, McLennan (1987), investigando a mesma relação em uma população de estudantes, encontrou relações significativas entre depressão e seis das onze crenças irracionais.

$\mathrm{Na}$ literatura científica investigada, não foram encontrados estudos que descrevessem as interações entre satisfação de vida e crenças irracionais. O presente estudo mostrou que, ainda 
que significativa, a correlação entre esses dois elementos se mostrou fraca, o que pode se dever ao tamanho da amostra e/ou à sua característica não-clínica. Como esperado, essa interação ocorre em direção negativa, o que mostra que, quanto menos crenças irracionais uma pessoa pode ter, maior a possibilidade de ela estar satisfeita com sua vida. A relação entre tais elementos não foi alterada de forma significativa, mesmo quando consideradas as subamostras de valores extremos na EFN.

\section{Conclusões}

O conceito de crenças irracionais é tão rico e complexo que suscita a curiosidade científica a respeito de sua relação com diversas questões clínicas. A presença de crenças irracionais já foi avaliada em sua relação com outros transtornos, embora não sejam constantemente confirmadas (Mayhew \& Edelman, 1989). No presente estudo, depressão e crenças irracionais se mostraram relacionadas de forma forte, embora de modo negativo, o que significa que nem sempre pessoas com altos índices de sintomas depressivos apresentam um número considerável de crenças irracionais. Outro achado relevante para o estudo do desenvolvimento humano constatado é a presença das crenças irracionais em sua tendência a diminuir com a idade.

Como se pode constatar, os achados do presente estudo foram consistentes com pesquisas prévias (Ziegler \& Leslie, 2003; David \& cols., 2004; Malouff, \& cols., 1992) e com a teoria de A. Ellis. Cabe reiterar que a importância do estudo é, sobretudo, clínica, onde se trabalha para que as pessoas aceitem o fato de que possuem amplo controle sobre seu destino emocional e comportamental. É esse controle, que é feito a partir de treinamentos de racionalidade, que lhes permite determinar inclusive se elas serão perturbadas por suas crenças. Com base no reconhecimento e do desafio dessas crenças, os indivíduos podem adquirir atitudes realísticas e sensíveis a respeito daquilo que não é desejável, e geralmente têm o poder de mudar o seu sistema de crenças, tornando-o mais funcional e saudável, (Ellis, 2003).

Os objetivos da TREC não tratam apenas de mostrar ao cliente quais são suas filosofias irracionais específicas, o que pode ser validado pelos instrumentos de avaliação psicológica. Tratam também de mostrar como essas ideias foram construídas e mantidas, e de pensar de modo mais racional, no intuito de não gerar aflições, criando menos crenças disfuncionais no futuro (Ellis, 2003). É justamente por meio do debate com as crenças irracionais que elas podem ser superadas, como uma espécie de treinamento da mente, característica das terapias cognitivas. É mediante essa superação que se pode almejar uma melhor relação consigo mesmo, com os outros e com o futuro, melhorando a interpretação da vida e de seus eventos, e buscando sua qualidade.

É possível apontar algumas limitações do estudo. Embora os resultados possam ser considerados relevantes, até por ser a presente pesquisa uma das poucas no estudo das crenças irracionais na população brasileira, a utilização de uma amostra relativamente pequena, predominantemente feminina e não clínica pode representar um empecilho à generalização das conclusões. Assim, são sugeridas novas propostas de estudos das crenças irracionais, em especial com populações clínicas, bem como se sugere o desenvolvimento de estudos de avaliação da eficácia da psicoterapia, considerando o tratamento específico para minimizar as crenças irracionais.

\section{Referências}

David, D., Montgomery, G. H., Macavei, B. \& Bovbjerg, D. (2004). An empirical investigation of Albert Ellis' binary model of distress. Journal of Clinical Psychology, 61(4), 499-516.

Diener, E., Emmons, R., Larsen, R. \& Griffin, S. (1985). The satisfaction with life scale. Journal of Personality Assessment, 49, 91-95.

DiLorenzo, T., David, D. \& Montgomery, G. H. (2007). The interrelations between irrational cognitive process and distress in stressful academic settings. Personality and Individual Differences, 42, 765-776.

Ellis, A. (2003). Early theories and practices of rational emotive bahavior therapy and how they have been augmented and revised during the last three decades. Journal of rational-emotive \&o cognitive-behavior therapy, 21(3/4), 219-243.

Giacomoni, C. H. (2002). Bem-estar subjetivo infantil, conceito de felicidade e construção de instrumentos para avaliação. Tese de Doutorado. Porto Alegre: Universidade Federal do Rio Grande do Sul (UFRGS). 
80 Matta, A., Bizarro, L., Reppold, C. T. Crenças irracionais, ajustamento psicológico e satisfação de vida

Giacomoni, C. H. (2004). Bem estar subjetivo: em busca da qualidade de vida. Temas em Psicologia da SBP, 12(1), 43-50.

Giacomoni, C. H. \& Hutz, C. S. (1997). A mensuração do bem-estar subjetivo: escala de afeto positivo e negativo e escala de satisfação de vida [Resumos]. Em Sociedade Interamericana de Psicologia (Org.). Anais XXVI Congresso Interamericano de Psicologia ( $\mathrm{p}$. 313). São Paulo, SP: SIP.

Hart, K. E., Turner, S. H., Hittner, J. B., Cardozo, S. R. \& Paras, K. C. (1991). Life stress and anger: moderating effects of type a irrational beliefs. Personality and Individual Differences, 12(6), 557-560.

Hutz, C. 1. \& Nunes, C. H. S. (2001). Escala fatorial de ajustamento emocional/neuroticismo EFN. São Paulo: Casa do Psicólogo.

Lega, L. I. (2002). A terapia racional-emotiva: uma conversa com Albert Ellis. In V. Caballo (Ed.). Manual de técnicas de terapia e modificação do comportamento. São Paulo: Santos.

Malouff, J. M., \& Schutte, N. S. (1986). Development and validation of a measure of irrational belief. Journal of Consulting and Clinical Psychology, 54(6), 860-862.

Malouff, J. M., Schutte, N. S. \& McClelland, T. (1992). Examination of the relationship between irrational beliefs and state anxiety. Personality and Individual Differences, 13(4), 451456.

McLennan, J. P. (1987). Journal of Clinical Psychology, 43(1) 89-91.

Marcotte, D. (1996). Irrational beliefs and depression in adolescence. Adolescence 31(124) 935-954.

Mayhew, R. \& Edelman, R. J. (1989). Self-esteem, irrational beliefs and coping strategies in relation to eating problems in a non-clinical population. Personality and Individual Differences, 10(5), 581-584.

Newmark, C. S., Frerking, R. A., Cook, L. \& Newmark, L. (1973). Endorsement of Ellis' irrational beliefs as a function of psychopathology. Journal of Clinical Psychology, 29, 300-302.

Rangé, B. (2001). Terapia racional-emotivocomportamental. Em B. Rangé (Ed.).
Psicoterapias Cognitivo-Comportamentais: um diálogo com a psiquiatria. Porto Alegre: Artmed.

Rangé, B., \& Fenster, D. (2004). Terapia racional emotivo-comportamental. Em P. Knapp (Ed.). Terapia Cognitivo-Comportamental na Prática Psiquiátrica. Porto Alegre: Artmed.

Shorkey, C. T. \& Whiteman, V. L. (1977). Development of the Rational Behavior Inventory: Initial validity and reliability. Educational and Psychological Measurement, 37, 527-534.

Warren, R., Zgourides, G. \& Jones, A. (1989). Cognitive bias and irrational belief as predictors of avoidance. Behaviour Research and Therapy, 27(2), 181-188.

Watson, P. J., Sherbak, J. \& Morris, R. J. (1998). Irrational beliefs, individualism-collectivism and adjustment. Personality and Individual Differences, 24(2), 173-179.

Yoshida, E. M. P. \& Colugnati, F. A. B. (2002). Questionário de Crenças Irracionais e Escala de Crenças Irracionais: propriedades psicométricas. Psicologia: Reflexão e Crítica, 15(2), 437-445.

Ziegler, D. J. \& Leslie, Y. M. (2003). A test of the ABC model underlying rational emotive behavior therapy. Psychological Reports, 92(1), 235-240.

Ziegler, D. J. \& Smith, P. N. (2004). Anger and the ABC model underlying rational-emotive behavior therapy. Psychological Reports, 94(3), 1009-1014.

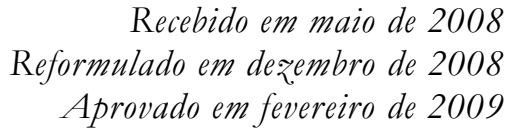


Sobre as autoras:

Adriana da Matta é psicóloga clínica formada pela PUCRS, mestra em Psicologia Clínica/PUCRS e Especialista em Terapia Cognitivo-comportamental/UFRGS. Atualmente cursa Doutorado no Programa de Pós-Graduação em Psicologia da UFRGS, desenvolvendo seu trabalho junto ao Laboratório de Psicologia Experimental, Neurociências e Comportamento.

Lisiane Bizarro é psicóloga, PhD em Psicologia pelo Institute of Psychiatry, King's College of London. É professora da Universidade Federal do Rio Grande do Sul (UFRGS) e Visiting Lecturer no Institute of Psychiatry King's College of London. É coordenadora pedagógica do curso de especialização em Terapia Cognitiva e Comportamental do Instituto de Psicologia da UFRGS e professora orientadora do Programa de Pós-graduação em Psicologia e do Programa de Pós-graduação na UFRGS.

Caroline Tozzi Reppold é doutora em Psicologia/UFRGS e professora adjunto da Universidade Federal de Ciências da Saúde de Porto Alegre/UFCSPA. 